\title{
Laparoscopic Repair of Obturator Hernia
}

\author{
Shen-Lin Wang, MD, Kung-Chen Ho, MD, Chuan-Yen Wei, MD, Shih-Ping Cheng, MD, PhD, \\ Tsang-Pai Liu, MD
}

Department of Surgery, Mackay Memorial Hospital and Mackay Medical College, Taipei, Taiwan (Drs Wang, Ho, Wei, Cheng, and Liu); Mackay Junior College of Medicine, Nursing, and Management, Taipei, Taiwan (Drs Cheng and Liu).

\begin{abstract}
Obturator hernias are rare, and most cases present as incarcerated hernias. They can be repaired either transabdominally or extraperitoneally, by the conventional open method or a laparoscopic approach. We report our technique of transabdominal laparoscopic direct repair using a running barbed suture in 2 cases. Old age and bowel distention are not contraindications to laparoscopic repair.
\end{abstract}

Key Words: Barbed suture, Laparoscopy, Obturator hernia.

Citation Wang S-L, Ho K-C, Wei C-Y, Cheng S-P, Liu T-P. Laparoscopic repair of obturator hernia. CRSLS e2014.00168. DOI: 10.4293/CRSLS.2014.00168.

Copyright (C) 2014 SLS This is an open-access article distributed under the terms of the Creative Commons Attribution-Noncommercial-ShareAlike 3.0 Unported license, which permits unrestricted noncommercial use, distribution, and reproduction in any medium, provided the original author and source are credited.

Address correspondence to: Tsang-Pai Liu, MD, Mackay Memorial Hospital and Mackay Medical College, Department of Surgery, No. 92, Zhongshan N Road, Section 2, Taipei 10449, Taiwan. Telephone: +886-2-25433535, fax: +886-2-27233897, E-mail: liutp@mmh.org.tw

\section{INTRODUCTION}

Obturator hernia is a rare condition, occurring at a population frequency of $0.073 \% .^{1}$ Most patients are thin, elderly women presenting with colic pain, ileus, and small bowel obstruction. The diagnosis of an obturator hernia is difficult because the symptoms are nonspecific, and the physical examination is rarely helpful. The pathognomonic Howship-Romberg sign, characterized by pain radiating down the medial aspect of the thigh on internal rotation of the hip, is present in fewer than half of patients and is often overlooked in the geriatric population. Delayed diagnosis may lead to significant morbidity and mortality because of bowel strangulation.

Once the diagnosis is made, obturator hernia should be surgically repaired. Several access routes and conventional repair techniques have been described. ${ }^{2}$ Recently, the number of reports using the laparoscopic approach has been increasing. Most laparoscopic techniques reported in the literature are transabdominal preperitoneal or totally extraperitoneal mesh repairs. ${ }^{3}$ Here, we present our technique of transabdominal laparoscopic direct suturing repair in 2 cases.

\section{CASE DESCRIPTIONS}

\section{Case 1}

A 90-year-old woman presented with nausea, vomiting, abdominal fullness, and constipation for 3 days. Physical examination revealed diffuse abdominal distension with decreased bowel sounds. Abdominal computed tomography showed a left obturator hernia and remarkable small bowel obstruction (Figure 1). The patient was brought to the operating room and made comfortable in a supine position. General anesthesia was induced. A Hasson's cannula was placed in the periumbilical region, and the abdomen was insufflated with carbon dioxide to a pressure of $15 \mathrm{~mm} \mathrm{Hg}$. Two additional trocars were placed at bilateral McBurney's points. The patient position was switched to the Trendelenburg position. Laparoscopic evaluation showed a segment of ileum incarcerated in the left obturator foramen with intestinal dilatation proximal to the incarceration (Figure 2). There was no evidence of a concomitant direct, indirect, or femoral hernia. The incarcerated bowel was gently reduced. After a period of observation, the bowel segment was noted to be viable, and resection was not necessary. The defect was closed using a running V-Lock (Covidien, Mansfield, Massachusetts) barbed suture (Figure 3). The total operative time was 45 minutes. The patient made an uneventful recovery and was discharged on the ninth postoperative day after her ileus resolved.

\section{Case 2}

An 83-year-old woman presented to our hospital for abdominal pain and constipation lasting 2 days. Physical 


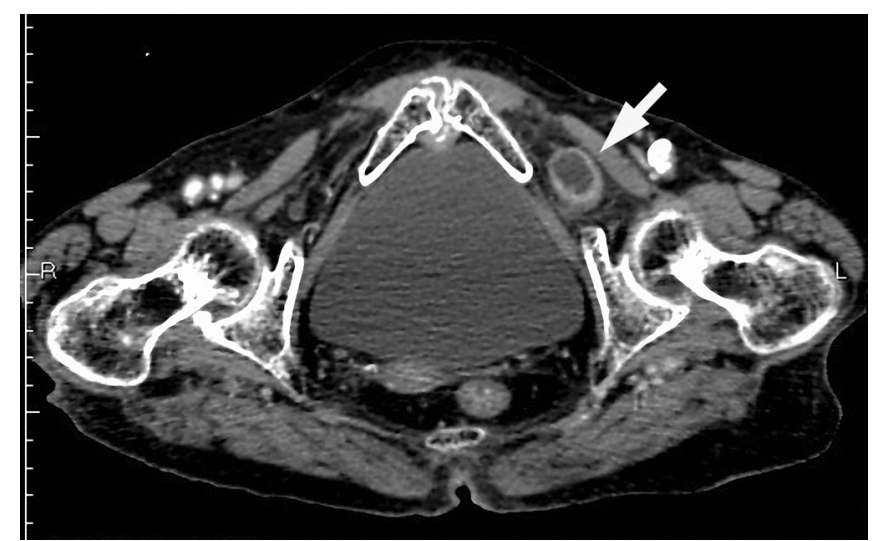

Figure 1. Computed tomographic scan of the pelvis showing a soft tissue shadow (arrow) between the external obturator muscle and pectineal muscle.

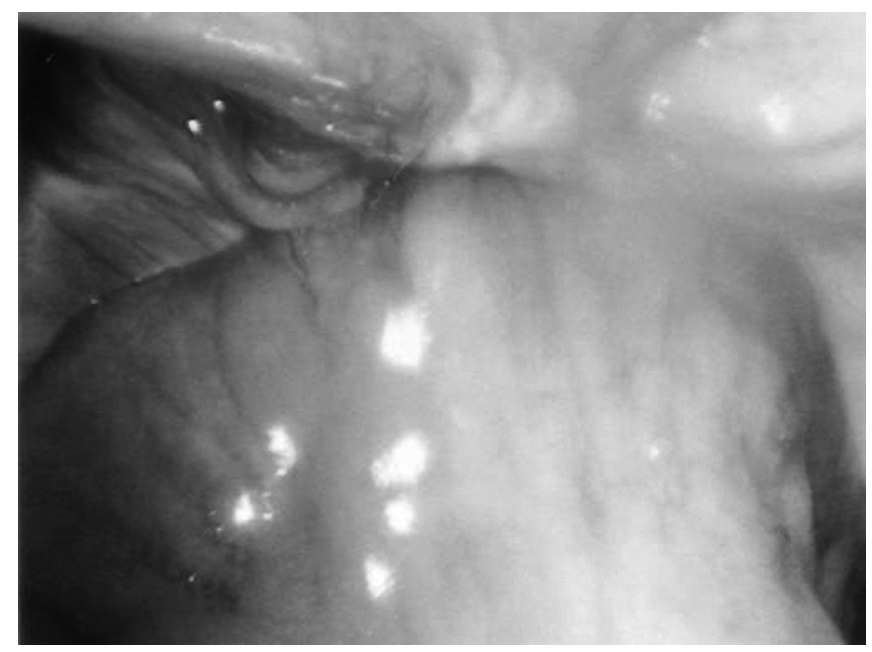

Figure 2. Intraperitoneal view a segment of small bowel incarcerated in the left obturator foramen.

examination revealed abdominal distention and epigastric tenderness. Abdominal computed tomography showed a right obturator hernia with intestinal obstruction (Figure 4). Using the technique as described above, transabdominal laparoscopic direct suturing repair was performed. Bowel resection was not needed. The total operative time was 62 minutes. Her postoperative recovery was uneventful. The patient was discharged on the eighth postoperative day.

\section{DISCUSSION}

The obturator canal is the opening in the superior part of the obturator membrane covering the foramen formed by the pubic bone and ischium, through which the obturator nerve, artery, and vein pass from the pelvic cavity

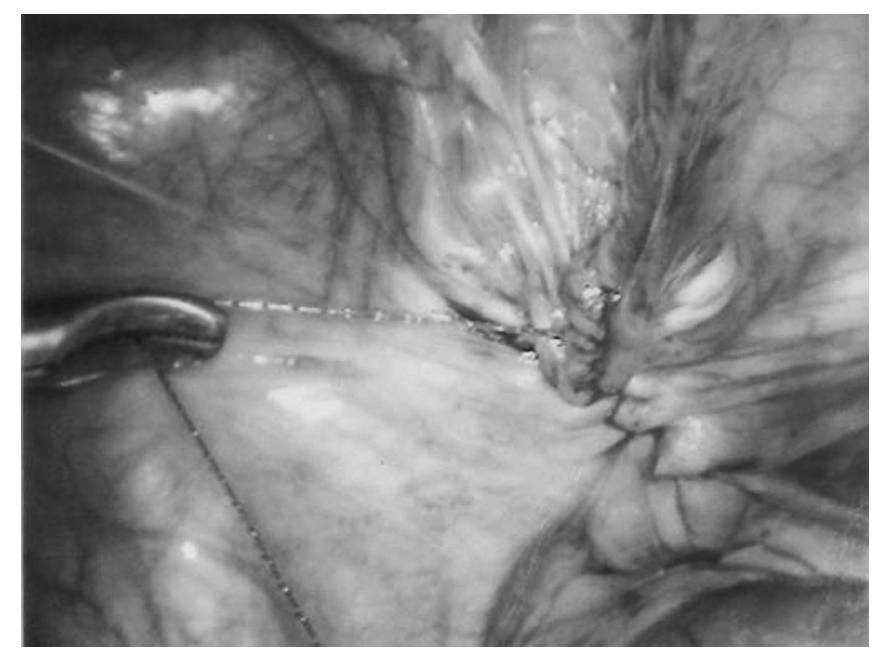

Figure 3. Intraperitoneal view after completion of the direct repair of obturator hernia.

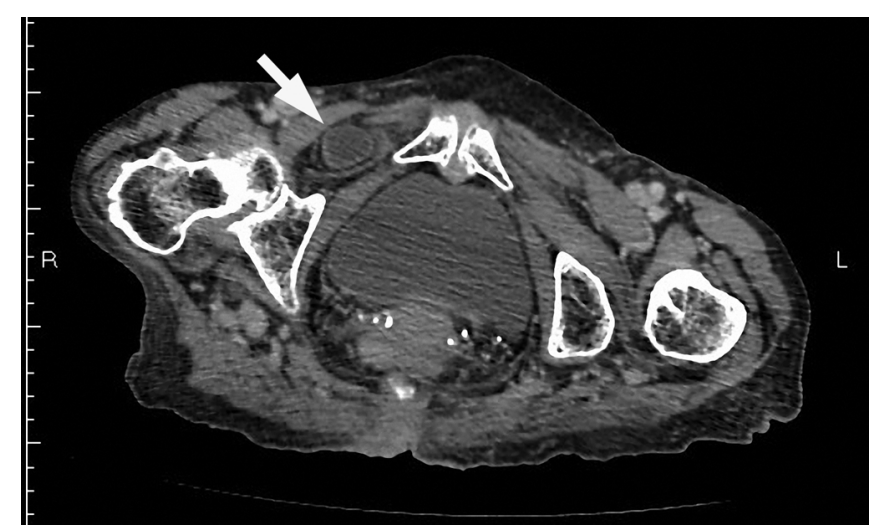

Figure 4. Computed tomographic scan of the pelvis showing the herniated loop (arrow) between the pectineus and obturator externus muscle.

into the thigh. The hernia is located deeply between the pectineus and adductor longus muscles. When the diagnosis is promptly made and strangulation is not suspected, it is suggested that the preperitoneal approach provides direct access to the hernia. ${ }^{2}$ When the diagnosis is unclear or when strangulation is suspected, the transabdominal approach is preferred. In our experience, the transabdominal approach permits the best assessment of intra-abdominal pathology and facilitates bowel resection in the cases of strangulated or perforated bowel.

For hernia reduction, an incision of the obturator membrane with care to avoid injury to the obturator nerve and vessels is sometimes required. Although the hernia orifice is usually small, direct primary suture repair is technically demanding because the foramen is deeply located and 
bordered by bone and tough obturator membrane. Simple peritoneal closure alone can result in hernia recurrence. The published literature suggests that a large prosthetic mesh in the preperitoneal space may be helpful to cover the obturator orifice as well as the femoral and inguinal areas. ${ }^{4}$ However, placement of a synthetic mesh carries a significant risk for infection when gross contamination is present or bowel resection is necessary. Moreover, mesh herniorrhaphy in the obturator canal may result in obturator neuralgia. We prefer direct suturing repair whenever possible.

Intestinal obstruction, commonly seen in patients with incarcerated obturator hernia, may make the laparoscopic approach difficult. In a recent review of case series, $71 \%$ of the elective cases could be treated successfully with a laparoscopic approach. ${ }^{3}$ Careful positioning and skilled suture techniques are imperative to repair obturator hernia laparoscopically. Although patients with obturator hernias are typically older, institutionalized or debilitated subjects with concurrent medical conditions, years of successful laparoscopic procedures in elderly patients have greatly reduced concerns of pneumoperitoneum-related complications. 5 In our patients, hemodynamic stability was well maintained throughout the procedure. We believe that a minimally invasive approach may be associated with decreased postoperative pain and more rapid recovery. Furthermore, the laparoscopic approach helps complete the examination of bilateral femoral and obturator canals. Surgeon's expertise plays an important role in the use of laparoscopy to handle dilated bowel without causing iatrogenic injury. We also found that the V-Lock type of barbed suture allows a faster and correct placement of stitches and knot tying.
We must acknowledge limitations in our report. Postoperative follow-up was short; thus, long-term outcomes, including recurrence and neuralgia, need to be further assessed. Although we did not note a large amount of tension on the tissues involved, and repair with relaxing incisions was not necessary, theoretically a tension-free repair would minimize the risk for recurrence. Large prospective studies, or meta-analyses, are required to determine whether the recurrence rate would be higher in suture-based repairs than mesh-based reinforcement of the obturator hernia defect.

\section{CONCLUSIONS}

Laparoscopic repair of obturator hernia is a safe and feasible method even in urgent settings. Old age and bowel distention are not contraindications to laparoscopic repair.

\section{References:}

1. Bjork KJ, Mucha P Jr, Cahill DR. Obturator hernia. Surg Gynecol Obstet. 1988;167(3):217-222.

2. Losanoff JE, Richman BW, Jones JW. Obturator hernia. J Am Coll Surg. 2002;194(5):657-663.

3. Deeba S, Purkayastha S, Darzi A, Zacharakis E. Obturator hernias: a review of the laparoscopic approach. J Minim Access Surg. 2011;7(4):201-204.

4. Skandalakis LJ, Androulakis J, Colborn GL, Skandalakis JE. Obturator hernia. Embryology, anatomy, and surgical applications. Surg Clin North Am. 2000;80(1):71-84.

5. Cheng SP, Yang TL, Jeng KS, Lee JJ, Liu TP, Liu CL. Perioperative care of the elderly. Int J Gerontol. 2007;1(2):89-97. 